\title{
Quantitative analysis of collagen content and amino acids in trabecular meshwork ${ }^{\star}$
}

\author{
Ian Finkelstein, Graham E Trope, Prasanta K Basu, Syed M Hasany, William S Hunter
}

\begin{abstract}
The purpose of this study was to compare collagen content in the $T M$ of normal and glaucomatous eyes, and to establish whether collagen levels change with age. Collagen content was measured in 30 normal and 27 age matched glaucoma trabeculectomy specimens by the sirius red dye binding technique, and in 14 normal and 15 age matched glaucoma specimens by amino acid analysis. Both dye binding data and amino acid analysis showed no statistical difference between normal and glaucoma samples. Age had no significant effect on mean optical densities or on the collagen-specific amino acids proline, hydroxyproline, and hydroxylysine. Amino acid variability, however, was statistically different between the two groups. These results indicate that mean collagen levels in the trabecular meshwork of glaucomatous eyes do not differ from those in normal eyes.
\end{abstract}

The cause of decreased outflow facility in primary open-angle glaucoma (POAG) remains controversial. However, abnormal trabecular meshwork (TM) collagen levels have been implicated by some authors. ${ }^{1}$ Changes in hydroxyproline concentration have been reported in the serum and urine of glaucoma patients. ${ }^{2}$ In comparison with normal samples increased levels of type I collagen have been reported in glaucomatous samples of human TM. ${ }^{3}$ An overall decrease in collagen types I, III, IV, and V has also been reported in glaucomatous eyes. ${ }^{4}$ However, other workers have reported no difference in collagen-specific amino acids in the TM of glaucomatous and control eyes. ${ }^{5}$ A marked increase of lattice ('curly') collagen and elastic material has been reported in the TM of eyes of older people. ${ }^{6}$ Age related increases in type I and IV collagen have also been reported in normal TM. ${ }^{7}$ The purpose of this study was two-fold: (1) to compare collagen levels in glaucomatous and normal TM, and (2) to determine whether age related changes occur in either group.

\section{Materials and methods}

Thirty normal and 27 age matched glaucoma trabeculectomy specimens from patients ranging in age from 39 to 90 years were obtained from fresh donor eyes and surgical trabeculectomies. All donor eyes and TM specimens were fixed in $10 \%$ buffered formalin (Fischer Scientific Co, Fairlawn, NJ, USA) within 12 hours of enucleation. Trabeculectomy specimens were excised under a surgical microscope by Watson's method of trabeculectomy. ${ }^{8}$ Each block of tissue was $2 \times 2 \mathrm{~mm}$ which contained TM plus a small

amount of sclera and cornea.
DYE BINDING STUDY

Tissue sections were processed and embedded in paraffin. Each tissue sample was analysed separately three times, with $2010 \mu \mathrm{m}$ sections being used per trial. Dry tissue weights were measured on an electrical balance (Mettler AJ100) prior to staining. The sections were placed on glass slides, deparaffinised, and hydrated in xylene, $100 \%, 80 \%$, and $50 \%$ ethanol and distilled water, each for intervals of 4 minutes. Tissue sections were immersed in Coplin jars containing $0 \cdot 1 \%$ sirius red dye (Chroma-Gesellschaft, Stuttgart, Germany) in saturated picric acid (BDH Chemicals, Toronto, Canada). The sections were incubated at room temperature and shaken for 30 minutes. Unbound dye was discarded by repeated rinsings of saturated picric acid and distilled water. The bound dye was eluted from the tissue by scraping the sections into a solution of $0.1 \mathrm{~N} \mathrm{NaOH}$ in absolute methanol (1:1, v:v), and allowing it to stand for 30 minutes at room temperature. After centrifugation at $4000 \mathrm{rpm}$ the supernatant was removed and optical densities (OD) were measured on a spectrophotometer (Gilford 2400) at $540 \mathrm{~nm}$. This technique was modified from that used by Yue et al. ${ }^{9}$

\section{AMINO ACID ANALYSIS}

Fourteen normal and 15 age matched glaucoma TM specimens were obtained by the methods outlined above. Each tissue sample consisting of $2010 \mu \mathrm{m}$ sections was deparaffinised and hydrated, and then scraped into separate test tubes. The samples were then freeze dried in a lypholyser at $48 \mathrm{~atm}\left(4850 \mathrm{kN} / \mathrm{m}^{2}\right)$ and $-50^{\circ} \mathrm{C}$, and dry weights were determined. Samples were hydrolysed with $6 \mathrm{~N} \mathrm{HCl}$ in vacuo at $106^{\circ} \mathrm{C}$ for 24 hours, and then dried and redissolved with 300 $\mu \mathrm{l}$ of doubly distilled water. Collagen specific amino acids (proline, hydroxyproline, and hydroxylysine) were measured in $\mathrm{nmol} / \mathrm{mg}$ of dried tissue on an amino acid analyser (Beckman $121 \mathrm{M}$ ).

Dye binding data were analysed by an $\mathrm{F}$ test to compare mean optical densities between groups that were controlled for age. Group differences in mean amino acid concentrations were also measured with the same test statistic. The effects of age on optical densities and amino acid levels for both groups were also analysed by an $F$ test.

\section{Results}

Results from the dye binding study showed mean optical densities of 0.525 (SEM 0.036) and 0.413 (SEM 0.045) for normal and glaucoma groups respectively (Fig 1). There was no statistical difference in mean optical density
Presented in part at Vision and

Ophthalmology, May

1989, Sarasota, Florida 


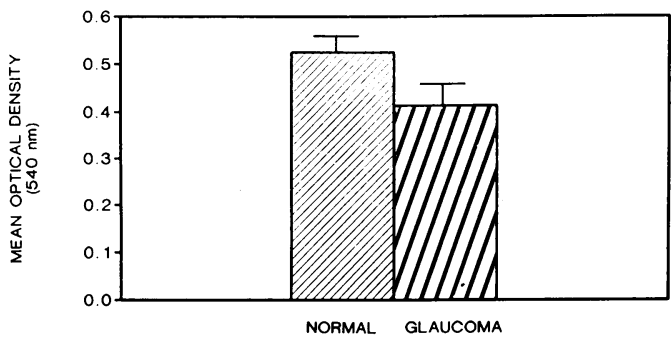

Figure 1: Dye binding data illustrating mean optical densities (with SEM) for normal and glaucoma TM samples.

between these groups. The change in optical density in the glaucoma group showed a slight increase with age, whereas the optical densities of normal eyes were virtually constant with increasing age (Fig 2). The difference between both groups, however, was not statistically significant.

Mean concentrations for all three amino acids were slightly lower for the glaucoma group, but the differences were not statistically significant. The mean hydroxyproline concentration for normals was 68.68 (SEM 12.56$) \mathrm{nmol} / \mathrm{mg}$ as compared to 63.03 (SEM 11.76) $\mathrm{nmol} / \mathrm{mg}$ for glaucoma samples. The mean proline concentration was slightly lower for normal and glaucoma groups at $65 \cdot 16($ SEM $10 \cdot 33) \mathrm{nmol} / \mathrm{mg}$ and $60 \cdot 74$ (SEM 10.28) $\mathrm{nmol} / \mathrm{mg}$ respectively. The mean concentration for hydroxylysine was $4 \cdot 15$ (SEM $0.77) \mathrm{nmol} / \mathrm{mg}$ for normal samples as compared with 3.61 (SEM 0.64$) \mathrm{nmol} / \mathrm{mg}$ for glaucoma samples (Fig 3). Amino acid concentrations appeared to decrease slightly with increasing age for both normal and glaucoma groups. However, the differences in amino acid concentration as a function of age were not statistically significant. Hydroxyproline is shown as a representative example (Fig 4).

Comparison of variations of amino acid concentration revealed more variable hydroxyproline, proline, and hydroxylysine concentrations in the glaucoma group than in the normal persons as a function of age. The probabilities of the statistical differences in variability between the two groups were: hydroxyproline $(\mathrm{p}=0.001)$, proline $(\mathrm{p}=0.002)$, and hydroxylysine $(\mathrm{p}=0.0001)$.

\section{Discussion}

Ten genetically distinct types of collagen have been described in mammalian tissues. ${ }^{10}$ Type I collagen constitutes the major collagen fraction in the cornea and sclera, while type III is generally found to coexist with type I. ${ }^{2}$ Immunohistochemical studies and amino acid analyses

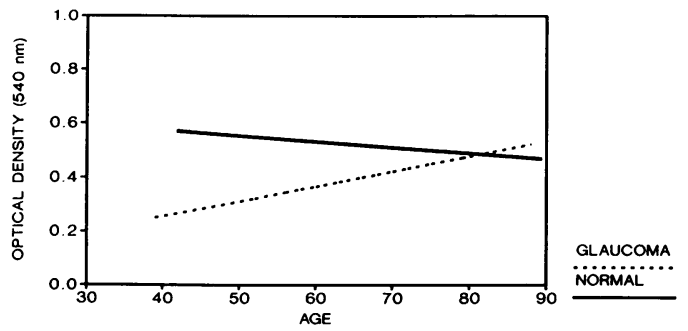

Figure 2: Effect of age on optical densities for normal and glaucoma TM samples.

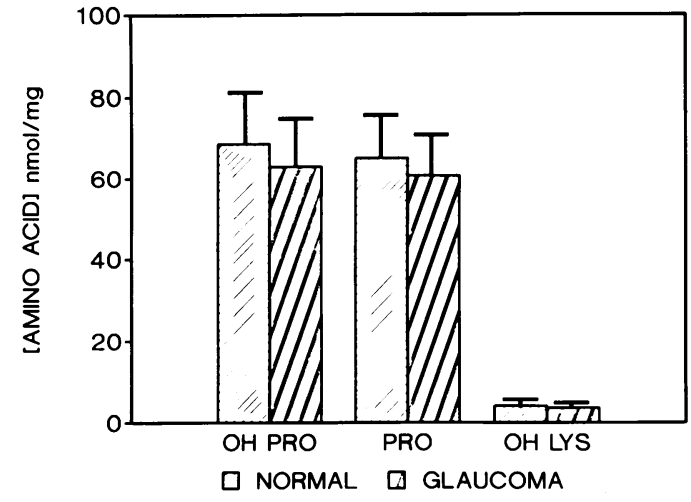

Figure 3: Mean concentration (nmol/mg) (with SEM) of hydroxyproline (OH PRO), proline (PRO), and hydroxylysine (OH LYS) for normal and glaucoma TM samples. Four values were treated as missing.

have detected types I, III, and IV in the trabecular meshwork and lamina cribrosa. ${ }^{11-13}$

Histochemical methods have been used by others to estimate collagen concentrations in ocular tissue sections prepared from formalinfixed, paraffin-embedded samples.' Sirius red dye is known to bind collagens preferentially to other proteins, specifically types I, II, and III. ${ }^{1+}$ This dye binding method provides a quantitative measurement of collagen content where the bound dye is proportional to the total amount of collagen present. Our results showed no statistical difference between mean optical densities of normal and glaucoma eyes, suggesting no difference in the amounts of collagen types I and III. These results differ from the report of Millard $e t$ al of increased levels of type I collagen in glaucomatous TM. ${ }^{3}$ They evaluated collagen levels using silver staining techniques and sodium dodecyl sulphate polyacrylamide electrophoresis. However, since we did not selectively test for type I collagen, there is a possibility that other collagen types may have masked the total quantitation of type I collagen.

Although we found no differences in amounts of collagen types I, III, and IV in the trabecular meshwork of normal and glaucoma eyes, Conner et al have recently reported at least a $50 \%$ decrease of collagen types III and IV in glaucomatous eyes and a $46 \%$ decrease of type $I$ in glaucomatous eyes. ${ }^{4}$ One possible explanation for our results includes the fact that we used trabecular meshwork samples derived from trabeculectomy specimens. Such samples have two drawbacks: firstly, they are always contami-

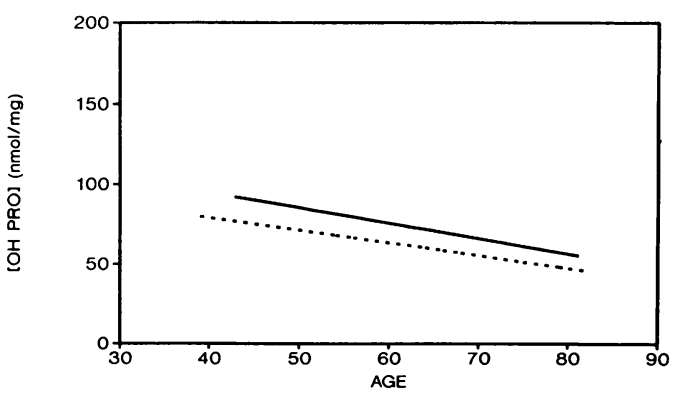

Figure 4: Representative illustration of the effect of age on amino acid concentration (nmol/mg). The concentrations for hydroxyproline (OH PRO) as a function of age are shown. Four values were treated as missing. Glaucoma ........ Normal 
nated by small amounts of cornea and sclera; and, secondly, the possibility exists that changes that occur in glaucoma, if any, could be masked by the small sample size of TM from the specimens. However, as trabecular meshwork specimens were the only source of glaucoma specimens for this study, such drawbacks were unavoidable.

Amino acid analysis is a well recognised method of determining tissue collagen concentrations. ${ }^{5713}$ The results of our amino acid analyses indicated no difference in mean concentrations of proline, hydroxyproline, and hydroxylysine in normal and glaucoma eyes, confirming the dye binding data.

Results from both parts of this study indicated that age related effects on collagen concentrations do not exist in either normal or glaucoma eyes in relation to the specific collagen types and amino acids that were tested. Although we found no differences in mean amino acid concentrations in both groups, significant differences were found in the variances of both groups, especially with hydroxylysine.

The significance of this finding is not known. A larger study on amino acid levels from normal and glaucoma trabecular meshwork may clarify this finding. For all three amino acids measured, normal samples showed greater variability than glaucoma samples. Using amino acid analysis, Horstmann $e t a l^{7}$ reported a rough estimate of the distribution of collagen types in TM based on 3-hydroxyproline/4-hydroxyproline ratios. These authors found age related increases in collagen types I ( $55 \%$ to $70 \%)$ and IV (2\% to $5 \%)$. These age related increases were slight but not statistically significant. In this respect the two studies are similar.

In conclusion, collagen concentrations as measured by dye binding and amino acid analysis showed no difference between normal and glaucomatous eyes. Amino acid levels in normal samples, however, were much more variable than glaucoma samples. Age had no effect on collagen levels in either group. Abnormally high or low trabecular meshwork collagen levels in glaucomatous eyes therefore do not appear to be the cause of decreased outflow facility in primary open-angle glaucoma.

Donated eyes and specimens from surgical trabeculectomies were kindly provided by the Eye Bank of Canada (Ontario Division), Oregon Lions Eye Bank, and the Ophthalmic Pathology Laboratory (Toronto, Canada), respectively. Statistical analysis was supervised by Professor M Chipman, Clinical Research Support Unit, Department of Preventive Medicine and Biostatistics, University of Toronto, Toronto, Canada.

1 Tengroth B, Rehnberg M, Ammitzböll T. A comparative analysis of the collagen type and distribution in the trabecular meshwork, sclera, lamina cribrosa and the optic nerve in the human eye. Acta Ophthalmol (Kbh) 1985; 63: 91-3.

2 Eroshevski T, Sviatkovskaja T. Collagen metabolism in primary open angle glaucoma. Oftalmol $Z h$ 1979; 34: 21-5.

3 Millard CB, Tripathi RC, Tripathi BJ, Vela A. Protein profiles and age-related changes in human trabecular meshwork. Invest Ophthalmol Vis Sci 1987; 28 (suppl): 131.

4 Conner LM, Cantor LB, Blevins CE, Schmedtje JF, Jersild RA. Quantification of collagen types in human trabecular meshwork in normal and glaucomatous eyes using immunomeshwork in normal and glaucomatous eyes using immuno(suppl): 223 .

5 Tengroth $B$, Ammitzböll T. Changes in the content and composition of collagen in the glaucomatous eye: Basis for a new hypothesis for the genesis of chronic open angl glaucoma. Acta Ophthalmol (Kbh) 1984; 62: 999-1008.

6 Rohen J, Lutjen-Drecoll E. Age changes of the trabecula meshwork in human and monkey eyes. Altern und Entwicklung 1971; 1: 1-35

7 Horstmann H-J, Rohen JW, Sames K. Age-related changes in the composition of proteins in the trabecular meshwork of the human eye. Mech Ageing Dev 1983; 21: 121-36.

8 Watson P. Trabeculectomy, a modified ab externo technique. Ann Ophthalmol 1970; 5: 199-205.

9 Yue BYJT, Sugar J, Schrode K. Collagen staining in corneal tissues. Curr Eye Res 1986; 5: 559-64.

10 Bailey AJ. Structure, function and ageing of the collagens of the eye. Eye 1987; 1: 175-83.

11 Stocker S, Grumaud J-A, Druguet M, Bernard M, Durand L. Collagène trabeculaire cornéoscleral. F Fr Ophtalmol 1980 3: 415-22.

12 Konomi H, Hayashi T, Sano J, et al. Immunohistochemica localization of type I, III and IV collagens in the sclera and choroid of bovine, rat, and normal and pathological human eyes. Biomed Res 1983; 4: 451-8.

13 Rehnberg M, Ammitzböll T, Tengroth B. Collagen distribution in the lamina cribrosa and the trabecular meshwork of the human eye. Brf Ophthalmol 1987; 71: 886-92.

14 Junqueira LCU, Bignolas G, Brentani RR. A simple and sensitive method for the quantitative estimation of collagen. Anal Biochem 1979; 94: 96-9. 